\title{
ASPECTOS ÉTICOS PARA LA IMPLEMENTACIÓN DEL EXPEDIENTE DIGITAL EN SALUD POR PARTE DE LA CAJA COSTARRICENSE DE SEGURO SOCIAL
}

\section{ETHICAL ISSUES CONCERNING THE IMPLEMENTATION OF THE ELECTRONIC HEALTH RECORD IN THE COSTA RICAN PUBLIC HEALTHCARE SYSTEM}

\author{
Allan González Estrada \\ Universidad Nacional, Costa Rica \\ allanarturo@gmail.com
}

Recibido: 15 de agosto / Aceptado: 30 de agosto / Publicado: 13 de noviembre

\begin{abstract}
Resumen
El rápido desarrollo de tecnologías de información, tales como servidores, bases de datos, dispositivos de almacenamiento, lenguajes de programación, plataformas de internet, ha hecho posible en los últimos años la implementación de la llamada "medicina en línea", la cual ha llevado a la creación del Expediente Electrónico en Salud. En Costa Rica, este fenómeno es reciente, y su ejecución "in-house" ha sido todo un reto para la Caja Costarricense del Seguro Social (CCSS). Sin embargo, implementar este sistema no está exento de riesgos, tanto en el nivel tecnológico como ético. Este escrito, resultado de una investigación en dicho campo, se ocupará de los problemas de carácter ético que esto conlleva, si bien el aspecto técnico no está desligado de ello. Entre los problemas éticos se pueden mencionar los relativos a las relaciones usuario-médico, médico-gerencia, implementación tecnológica, preparación ética de profesionales en salud y de tecnologías de la información, aspectos relacionados con la seguridad y privacidad de la información, nivel de acceso a internet de la población, así como derechos humanos, participación y vigilancia ciudadana. Se expone el porqué de la necesidad de que los profesionales de salud e informática cuenten con preparación ética y que se discuta con ellos conceptos como autonomía, beneficencia y toma de decisiones éticas.

Palabras clave: filosofía de la tecnología, ética aplicada, sistema de salud, confidencialidad, beneficencia, datos digitales, medicina en línea, protección de datos
\end{abstract}

\begin{abstract}
:
The rapid developments of information technologies such as servers, data bases, storage devices, programming language, internet platforms, and so on has made the introduction of so-called "online medicine" possible in the last several years, leading to the creation of the Electronic Healthcare Record. In Costa Rica, the use of such technologies is recent, and their
\end{abstract}


implementation in-house has been quite challenging for the Costa Rican public healthcare system Caja Costarricense del Seguro social (CCSS). However, the use of this system is technologically as well as ethically risky. This article, which is the result of a research project in the aforementioned field, deals with the ensuing ethical issues, though the technological aspect is not disconnected from it. Some of the ethical problems that can be mentioned are those concerning the relationship between physicians and patients, or physicians and management, the technological implementation, ethical training for health care and information technology professionals, issues related to data security and privacy, internet coverage for the population, as well as human rights, participation and citizen vigilance. The necessity of health and information technology professionals receiving ethical training is presented, as well as the need to discuss with them concepts such as autonomy, public service and making ethical choices. Keywords: philosophy of technology, applied ethics, health system, confidentiality, digital data, in line medicine, data protection

\section{Introducción}

Cuando mi madre me llevaba de niño a la consulta médica, que me correspondía en la Clínica Marcial Fallas, en Desamparados, perteneciente a la Caja Costarricense de Seguro Social (CCSS), ${ }^{1}$ el médico verificaba algunos datos, dibujaba garabatos en un folder de manila y luego hacía preguntas varias, agregaba más garabatos y daba el diagnóstico. Luego nos entregaba unas recetas médicas escritas con más garabatos: unas inyecciones, un jarabe para la tos y unas pastillas azules con blanco. Eso era todo. Yo volvía en llanto a mi casa por el dolor de la inyección y por haber esperado al menos una hora, frente al mural de la entrada a la clínica ${ }^{2}$ y mi madre regresaba con la satisfacción de haber recibido una buena consulta médica y la esperanza de que mi tos y mi bronquitis desaparecieran gracias a la acción de aquel bulto de medicinas. No era, sin embargo, el único médico que me examinaba, por esa razón aquel folder contenía, a la corta edad de mis nueve años, un ya bastante nutrido historial médico ${ }^{3}$. En esas épocas, el manejo digital de la información era casi nulo, y la mayoría de las transacciones electrónicas se hacían por medio de las llamadas tarjetas perforadas. Pasaron aproximadamente 40 años para que las tecnologías de información, el desarrollo de computadoras, la mejora en los

1 En esa época también existían las unidades sanitarias a las que fui de visita un par de veces.

2 Pintado por el reconocido artista y Premio Magón Rafa Fernández, cuando en 1974 fue seleccionado para ejecutarlo. Lo tituló: "Alegoría al trabajo" y mide 45 × 2.5 metros.

3 En este sentido, el compartir información se vuelve algo fundamental y, por supuesto, dentro de un contexto y comunidad particular, por ejemplo, el sistema de salud. Desde la época de las pinturas rupestres, lo que está en la mente se pone en el mundo exterior para que otros puedan tener acceso a ese conocimiento. Sin embargo, en el caso de las profesiones médicas, el conocimiento de alguna manera se volvió más esotérico, en parte por 
lenguajes de programación, la llegada de la internet y el perfeccionamiento de las bases de datos permitieran la posibilidad de contar con una plataforma digital e integrada de la información médica. A esto es a lo que se llama hoy Expediente Digital Único en Salud (EDUS) ${ }^{4}$. Con anterioridad -como ya se mencionó- la información médica era, o es aún en algunos casos, documentada en papel para que pudiera ser consultada por otros profesionales del sector salud, o al menos esa era la intención original del expediente físico ${ }^{5}$. Sin embargo, esta forma de almacenar datos se volvía con el tiempo poco práctica y presentaba algunos problemas, por lo que la alternativa digital se ve ahora más simple y eficaz, tanto para los usuarios de los sistemas de salud como para los diferentes profesionales que laboran en ese sector ${ }^{6}$.

\section{Antecedentes del expediente digital en el mundo y en Costa Rica.}

El uso del expediente electrónico digital es más bien reciente. Fue el médico Lawrence Weed quien acuñó por primera vez el término "expediente electrónico” (http://healthtechnologyreview.com/art135_history_of_electronic_medical_records.php), en el marco de un proyecto de cooperación entre médicos y profesionales en cómputo de la Universidad de Vermont.

la forma de escribir y en parte, por el lenguaje técnico utilizado por el médico, que resulta difícil de comprender para el usuario.

4 La CCSS da una definición similar, sin embargo, es evidente el uso con fines administrativos de los datos médicos. De acuerdo con el reglamento del expediente digital único en salud, el sistema de información EDUS se define como "...un sistema de información desarrollado para facilitar la prestación de servicios de salud y apoyar la gestión clínica y administrativa. Está constituido por un programa informático o aplicativo que responde a los procesos y normas asociadas con la atención ambulatoria, hospitalaria, de urgencias..." (Reglamento, 2018, p. 5). De esta definición se tendría que explicar el uso de los datos, ya que no queda muy claro qué tipo de datos son los que cataloga la CCSS como datos médicos, sobre este punto se volverá más adelante.

5 Es de mencionar la idea de responsabilidad, por ahora definida como esa capacidad de tomar decisiones por parte de los usuarios del sistema de salud. Esto para que no solamente sean actores pasivos ante las decisiones del médico o profesionales de salud; en otras palabras, una de las ventajas de estas tecnologías es que ofrecen una herramienta para poder "empoderarse" no ya de los cuerpos -como intenta el gremio médico- sino para decidir sobre mejores tratamientos médicos. Estas tecnologías permiten un intercambio de ideas entre usuarios, a la vez que son una fuente de críticas a la idea del paternalismo médico. El expediente digital, al utilizar caracteres legibles evita, por un lado, el uso de la escritura médica a mano y, por otro, la posibilidad de obtener esa información de una manera clara. Esto lleva a una idea de responsabilidad más acabada sobre la salud (ver por ejemplo Beyond entitlement: the social obligations of citizenship, Mead L, 1968), tanto de los usuarios como de los profesionales de salud, que de alguna manera era lo que esperaba William Beveridge cuando sentó las bases históricas del estado benefactor en el Reino Unido, esta idea se retomará más adelante.

6 Y que si el software se compra a una compañía extranjera los costos podrían ser más bien elevados, la opción de la CCSS fue hacer uno propio o "in-house". 
Luego, otros proyectos como el $\operatorname{COSTAR}^{7}$, de la Universidad de Harvard, lo implementaron para el cuidado ambulatorio en los años 70, y le dieron con ello mayor estímulo al recurso de los expedientes digitales. Pero no fue hasta los 90 que este se popularizó.

En Costa Rica, el EDUS, implementado para el sector público ${ }^{8}$, inicia con la aprobación en primer debate, por parte de la Asamblea Legislativa, de la ley 17214 "Ley Expediente Digital único en Salud”, en el año 2012. De acuerdo con Varela, este sistema echa a andar con la idea de integrar toda la información del paciente en un reservorio electrónico:

En el 2011 la Asamblea Legislativa de la República de Costa Rica decretó la Ley Expediente Digital Único en Salud. El EDUS consiste en un expediente digital para registro y almacenamiento de la historia clínica de cada paciente de la CCSS, para el cual se ha planteado un alcance de la automatización de los registros clínicos de los pacientes en 1.014 Equipos Básicos de Atención Integral en Salud (EBAIS), 103 áreas de salud, 29 hospitales, 775 puestos de visita periódica, logrando la cobertura y beneficio de toda la población del país, cerca de 5 millones de usuarios finales. (Varela, 2017, p. 4)

Como puede notarse, la implementación del EDUS es de grandes proporciones, conlleva un gran esfuerzo tecnológico y logístico para capacitar al personal del sector salud y profesionales en el área de las llamadas tecnologías de información9. Ya para el 2015, el 30 \% de la población estaba cubierto por el EDUS, de acuerdo con el periódico El Financiero del 8 de febrero del 2015.

\section{Base ética del EDUS.}

Es importante mencionar los orígenes utilitaristas de la idea de cómo implementar el EDUS. De acuerdo con Giselle Barrantes, del departamento

7 Siglas de Computer Stored Ambulatory Record, un sistema desarrollado en 1968 por Octo Barnette y Jerome Grossman, de la división de ciencias de la computación de la Universidad de Harvard. Está aún en uso, basado en un lenguaje de programación llamado MUMPS. En sus primeros dos años llevaba ya el registro de 20000 pacientes. (http://www.clinfowiki.org/wiki/index.php/COmputer_STored_Ambulatory_Record_\%28COSTAR\%29)

8 En el sector privado, la clínica Bíblica inició con un proceso de expediente electrónico en el año 1995, por medio de un programa que permitía integrar información de los pacientes. Hasta el día de hoy alberga datos de rayos x, farmacia, entre otros (Bíblica, 2012, pp. 12-14), este se ha robustecido y ha sido parte del sistema integrado de gestión hospitalaria en el sector privado.

9 Es importante notar que no todas las personas aceptan por igual los programas informáticos; algunas solo usan, por ejemplo, el procesador de texto, cualquier otro les resulta una pesadilla. 
informático, durante una entrevista sostenida el martes 6 de marzo de 2018 en las oficinas centrales de la CCSS, las decisiones sobre los procesos de automatización fueron tomadas en búsqueda del mayor beneficio para la población: "toda la priorización de servicios responde en el EDUS al beneficio de la mayoría" (Barrantes, 2018, minuto 51.43). Sin embargo, lo primero que se desarrolló fue el agendamiento y la citación. Así, se puede notar el fuerte componente ético utilitarista que inspira la manera en la que opera el diseño del EDUS. Pero ¿por qué una ética utilitarista?, lo que sugiere un sesgo ético en la toma de decisiones. Dicho de otro modo, si utilidad también se puede definir en términos de consecuencias en los ámbitos económico y de eficiencia, ¿cómo se puede basar en esta ética el proceso de atender la salud a la población? La respuesta parece ser clara: el objetivo es optimizar recursos. Este, sin lugar a dudas, es un aspecto problemático realtivo a cómo se conceptualiza la ética del EDUS. En otras palabras, si la CCSS ve en el EDUS una fuente de información para mejorar el proceso de distribuir recursos, pero el beneficio al paciente se limita a que los médicos en diferentes lugares puedan ver la información en forma digital, entonces ese beneficio parece ser más bien secundario $^{10}$, o bien derivado de otras ideas alrededor del EDUS. Es un hecho que la población no parece haberse involucrado o haber exigido el manejo de datos adecuado por parte de la CCSS, y que simplemente ve el EDUS como una "mejora tecnológica"11. Tal como lo señala el informe GiSWatch (2014), el implementar el EDUS no es visto como un tema que tenga lugar en la sociedad civil para la discusión sobre el derecho a la salud, ni sobre el manejo de datos y las posibilidades que abre de ser también usado para la vigilancia ciudadana; mucho menos para exigir actualizaciones éticas a los profesionales de salud e informática para el manejo de datos (GiSWatch, 2014, párrafo 7).

\section{3. Ética y tecnologías de información.}

Dada su reciente llegada, y su origen como producto humano, las tecnologías de información deben ser un asunto reflexionado a nivel de ética. Estas en nuestros países responden, generalmente, a procesos de colonización

10 En este estado de la investigación, esta premisa parece una concluyente, sin embargo, aunque aún no lo sea, diferentes entrevistas lo dejan sentir así. Por ejemplo, cabe la pregunta: ¿cómo participa el paciente en el EDUS? ¿Solo como un actor cuyos datos se llenan en un formulario digital?

11 Estos datos se mostrarán más adelante conforme avance el proyecto. 
cultural. En otras palabras, hay una constante transferencia de tecnología desde los países más poderosos, pero una recepción pasiva de esta ${ }^{12}$ por parte de los receptores. Esto se hizo patente durante los debates de las últimas elecciones presidenciales en Costa Rica. Los diferentes candidatos insistieron en apostar al desarrollo a partir de la ciencia y la tecnología ${ }^{13}$, de ahí que sea casi imposible no pensar que esta es vista como una especie de bálsamo de Fierabrás, que soluciona todos nuestros problemas -incluidos los emocionales- ${ }^{14}$. Si esta fuera, en efecto, una maravilla que nos da la felicidad anhelada cabría preguntarse si es accesible para todos los costarricenses, o solo para algunos. Históricamente, a nuestros países ha llegado la tecnología que el "mundo desarrollado" ha querido enviarnos, y solo para algunas personas. Dadas las relaciones de poder, y nuestro lugar geopolítico, hemos absorbido la que se nos da, pero ¿ha sido esa la que se nos ha ofrecido?, sin estar diseñada para nuestras necesidades. En otras palabras, la acción ha sido forzar la tecnología para que funcione de acuerdo con algunas necesidades que no teníamos. Tal como lo menciona Manuel Arce, en su libro Visitas al desván, el problema es ético:

Inmersos en un orden impuesto por circunstancias fuera de control [sistema económico y político] los profesionales en computación raras veces se cuestionan este orden o reflexionan sobre los dilemas éticos que su profesión les impone subrepticiamente en el contexto del subdesarrollo... [la idea] de que la tecnología nos saca del subdesarrollo... De aquí se pasa a un estadio tan absurdo como grotesco: la tecnología llega a ser un fin, no un medio, y el ser humano medio, no fin. Los nuevos dioses se llaman "eficiencia", "producción", “desempeño" ... (Arenales, 2006, p. 22)

12 Aunque en Costa Rica ha existido un esfuerzo por atraer inversión de calidad que utilice el talento humano, no ha sido suficiente para lograr crear tecnología propia, el caso de Intel es un ejemplo. Se apostó al desarrollo de carreras tecnológicas y, dadas las características del sistema económico en el que estamos terminó con la transferencia a otro país de la parte de manufactura. Queda, sin embargo, un centro de diseño de microprocesadores, pero de eso, ¿qué conocimiento le queda al país?

13 Empleabilidad a partir de la ciencia y la tecnología es y ha sido últimamente la apuesta de algunos candidatos presidenciales tanto en Costa Rica como en América Latina.

14 Esto no es una cuestión baladí, las tecnologías de la información tienen un fuerte impacto en la salud emocional de las personas. 
La anterior cita es de vital importancia, puesto que descompone la idea de que la tecnología nos permita, de forma mágica, salir del subdesarrollo ${ }^{15}$, a todas luces es una falacia ya que, más bien, en nuestros países es recibida como algo confuso o divorciado de las dimensiones éticas y sociales. De lo anterior se pueden sugerir varias cosas:

1. Los problemas de las tecnologías de información en el nivel ético necesitan explorarse de acuerdo con la naturaleza de las necesidades tecnológicas de nuestros pueblos.

2. La falta de preparación ética de los profesionales en cómputo lleva a que manejen de manera "tecnologizada" nuestra información.

3. La idea de que la tecnología per se es la que nos lleva al desarrollo es una falacia.

4. Existe una visión utilitarista de la tecnología y de las personas.

5. Las personas, en un contexto que privilegia lo tecnológico, son vistas como fines y no como medios, violándose la tercera fórmula del imperativo categórico de Kant.

El contexto actual une pues dos disciplinas que podrían verse por completo separadas, salud e informática, pero con un actor en común: las personas o usuarios de los sistemas de salud. Estos están cercados por varios flancos: la medicina, la tecnología, el paternalismo médico, la falta de preparación ética, tanto de profesionales en salud como en computación, y la necesidades de un sistema capitalista que valora, más que nunca, la eficacia de los procesos productivos, en los cuales, por ejemplo, la atención de personas por parte de los profesionales de salud de la CCSS en sus tres niveles está bajo un escrutinio constante por parte de las jefaturas, más que nada en nombre de la eficacia y la producción. Por ello, los problemas éticos en el sector salud son más bien complejos y, como se mencionó, no son tan simples como hace 70 años, hay una transdiciplinariedad que impacta de forma directa a las personas.

15 Existen, no obstante, procesos de apropiación tecnológica, no podemos como "países subdesarrollados" partir de cero, tal como lo hizo Japón después de la Segunda Guerra Mundial. 


\section{Problemas con la base ética del EDUS.}

Al tener en cuenta esos dos campos, cuyo nexo parece ser poco explorado, surgen algunas preguntas: ¿Cuál es la mejor teoría de justicia que puede aplicarse en un mundo delineado por las teorías de información (internet y programas informáticos)? ¿Cómo aplicar esa teoría de justicia de modo que pueda desembocar en una teoría ética para una sociedad tecnificada, que en la actualidad se basa en una visión utilitarista, automatizada y discriminatoria a la hora de la distribución de recursos? (Rawls, 1974, Norman Daniels, 1985). Dicho de otra manera, existe una necesidad imperiosa de encontrar un puente entre la teoría de la filosofía política, la ética y una doctrina de derechos humanos que garantice la igualdad de condiciones ante un sistema de salud implementado en una sociedad dependiente de la tecnología y enmarcada en una economía de mercado. En otras palabras, una teoría de justicia que contemple las necesidades de todos los ciudadanos y base en ellas la distribución de recursos. Una salida sería explorar la aplicación ética de la teoría de juegos de John Nash, que evidencia la necesidad de colaborar entre los actores más que la competencia para lograr un fin satisfactorio. Esto será contemplado en la segunda parte de la investigación.

Antes de continuar, es necesario contextualizar el uso de estas tecnologías $^{16}$ en salud y las ventajas que ofrece. Fabricio Picado señala que:

Como se indicó anteriormente, la combinación y el uso de las anteriores tecnologías [registro de salud electrónico, dispositivos móviles, telemedicina, drones] puede mejorar el acceso a través de los servicios de salud. Disponer de un registro electrónico va a permitir, en un corto plazo, que, a través de aplicaciones diseñadas para dispositivos móviles, los usuarios accedan al expediente electrónico que incluye su historial clínico o visualicen el resultado de un examen de laboratorio o del servicio de radiología. Ese acceso permitirá al usuario disponer de información sobre su condición de manera inmediata, disminuyendo tiempos en los servicios de salud, ya que se automatizan los componentes de atención. (Picado, 2014, p. 177)

16 De acuerdo con el consejo de Bioética de Nuffield, las tecnologías aplicadas en el sector salud son: 1) acceso en línea a la información de salud, 2) expedientes electrónicos 3) compras en línea de productos farmacéuticos 4) telemedicina y 5) exámenes predictivos: genética personal, perfiles e imágenes del cuerpo (Nuffield, 2010, p. 50). Por supuesto, cada uno de estos aspectos implica problemas éticos. 
Lo antepuesto deja ver el gran impacto que tiene la tecnología en la salud de las personas y en los respectivos servicios ${ }^{17}$. Pero esto se ubica en un marco ético utilitarista, sin una teoría de justicia específica. Aquí podemos encontrar un primer problema en el nivel ético. Si bien es cierto que la tecnología se nos presenta como una forma de automatizar el trabajo y mejorar tiempos de atención en el sector salud, no se contempla la forma en la que las personas pueden manejar ese sentido de responsabilidad, que puede conferir el conocimiento de sus datos médicos, tanto pacientes como profesionales, y su nivel de compromiso con las políticas de salud pública, como es el caso del EDUS. ¿Para qué serviría el conocer esa información si no hay una horizontalidad en la relación médico-usuario, y si no hay claridad del uso de los datos obtenidos por parte de los usuarios? Sin embargo, como ya se mencionó, la tendencia es que, por medio de las tecnologías de información, particularmente de la internet, se dé un mayor empoderamiento de las personas en relación con su cuerpo $^{18}$, y por ende, con las decisiones médicas ${ }^{19}$; el uso de tecnologías como el expediente digital podría generar una mayor autonomía en el paciente. Pero, dada la gran cantidad de datos que existe en internet, y al tenerse acceso a

17 En Costa Rica se han dado problemas con la tecnología en el uso médico, y quizás uno de las más recordados fue el de los irradiados en el hospital San Juan de Dios en 1996, cuando 115 personas fueron irradiadas en la unidad de cobalto (Co-60) Alcyon II. ¿Como se podía reflexionar éticamente sobre ese problema? Al menos con las herramientas académicas con las que contaba el país, un acercamiento por el principialismo (T. L. Beauchamp, J. F. Childress, 1979), el principio de precaución, que podría verse como un derivado de la ética aristotélica con el concepto de fronesis. Los trabajos de Hans Jonás sobre los imperativos éticos, y podría también aplicarse la reflexión de Arne Naes. Estos conceptos clásicos de la filosofía pueden aplicarse entonces a los problemas éticos asociados a tecnologías y desarrollos científicos, lo que ayudaría a un uso ético de esta y de la ciencia misma.

18 Por ejemplo, esta apropiación del cuerpo y de la información, tiene una vertiente en el consumismo -que también es uno de los principales responsables de la medicalización de la sociedad -y compañías como Google y Microsoft ofrecen sus servicios de expediente electrónico, pero hace especial hincapié en el control personal de los datos-, esto lleva a la pregunta de ¿quién es el dueño de los datos médicos, el hospital, en nuestro caso la CCSS, o la persona? En el caso de google, el eslogan publicitario es "usted siempre tiene el control" y "la información médica le pertenece solo a usted”, aunque el servicio de Google dejo de funcionar en el 2012, marcó una pauta en la información médica en internet. Microsoft, ofrece el servicio llamado "healthVault" al garantizar que la información siempre va a estar en línea (https://international.healthvault.com/cr/en), estos servicios aseguran que los datos no son compartidos con nadie más, lo que demuestra las preocupaciones existentes y asociadas a la privacidad de los datos médicos, que es, hoy por hoy, una mina de oro para las compañías farmacéuticas, por ejemplo. También, esta apropiación es fundamental en las teorías feministas, en otras palabras, los datos sobre control de natalidad y métodos reproductivos es de vital importancia a la hora de tomar decisiones éticas y el manejo de la sexualidad.

19 Sin embargo, esto es problemático, dado que no existen filtros sobre la información relacionada a enfermedades que existen en internet, como menciona el reporte del consejo de Nuffield, anteriormente las personas socializaban datos de salud (periódicos, revistas, familiares, vecinos, etc.) (Nuffield, 2010, p. 65), pero en la actualidad, para los que tienen internet, este se ha convertido en la mayor fuente de información. Sin embargo, esto es un inconveniente también, pues la primera línea de atención con el médico puede perderse, y al poder inclusive comprar medicamente en línea, se puede provocar un mal mayor, por lo que esto llevaría a un daño más que 
los de índole médica -que es limitada por parte del usuario- podría darse un problema de "cotejo de información", lo cual incidiría negativamente en la salud de las personas. De hecho, de acuerdo con el reporte "Britania Digital", cerca del $50 \%$ de los usuarios de internet la consideran el primer "puerto de llamada"

(https://www.gov.uk/government/uploads/system/uploads/attachment_data/file/228844/7650.pdf) para tratar de resolver problemas de salud, lo cual deja al médico como una segunda o tercera opción ${ }^{20}$. Los datos anteriores han servido para introducir parte de la problemática asociada a la puesta en práctica del expediente digital en salud en Costa Rica. Pasaremos ahora a analizar las características del sistema costarricense y cómo se integra el uso de las tecnologías de información.

\section{Desarrollo histórico de la medicina en Costa Rica y necesidad de la implementación del Expediente Digital Único en Salud.}

No es el objetivo de este apartado hacer un recorrido exhaustivo por los principales hechos históricos ${ }^{21}$ que llevaron a la existencia de la CCSS, más bien se trata aquí de una cierta cronología que nos explique el origen de la necesidad de constituir políticas dirigidas a la implementación de tecnologías en los servicios que esta institución brinda. Entre los años 1941 y 1943 se dan importantes cambios sociopolíticos que llevan a crear la CCSS, como la institución encargada de velar por la salud de los costarricenses. Sin embargo, los profesionales en esta área no se preparaban en el país. Si bien es cierto que la Universidad de Costa Rica abrió sus puertas en 1940, es en 1947 que inició la Escuela de Medicina. Luego, en el año 1953 se fundó la Facultad de Medicina y fue en 1961 que se abrió la primera promoción, que graduó a la primera generación en 1965. Es de vital importancia recordar que en 1947 se

un beneficio, razón por la cual existe una responsabilidad estatal en alguna medida para evitar el mayor mal posible a un población medicalizada, que puede consumir sin más las medicinas, sin la adecuada receta médica; a la larga, el costo económico de esto sería aún mayor, viéndolo desde una ética utilitarista.

20 Lamentablemente, en Costa Rica no hay datos sobre este tema; sin embargo, las mayores búsquedas en Google del año 2017, hechas por costarricenses ((https://trends.google.com/trends/yis/2017/CR/), están relacionadas con la salud, por ejemplo para saber qué es el lupus, cómo bajar de peso, y cómo quitar la caspa. Es probable que sean las respuestas halladas en Google las que sugieran diferentes medicamentos y no las consultas médicas realizadas de manera directa al profesional de salud.

21 Para esto puede consultarse el artículo del Sr. Guido Miranda "En el 75 aniversario de la CCSS" donde el autor hace una referencia histórica de cómo se conformó la institución. 
publicó el Código de Nuremberg ${ }^{22}$, el cual estableció los lineamientos éticos que debían seguirse en experimentos médicos con seres humanos. Si bien es cierto, que esos primeros años de desarrollo de la medicina en Costa Rica estuvieron orientados más bien hacia una prestación de servicios asistenciales ${ }^{23}$, de acuerdo con Daniel Bustos Montero, Costa Rica fue uno de los primeros países en preocuparse por la práctica ética de la profesión médica. Como lo indica en su artículo "Evolución de la Bioética en Costa Rica, una historia reciente":

.... Curiosamente, siendo un sistema fundamentalmente asistencial, y con poca cultura para la investigación, fue la bioética en investigación la que se desarrolló con más fuerza. En 1972, mucho antes de que se hiciera del conocimiento público el estudio Tuskegee, y el propio Informe Belmont en los Estados Unidos de América, el Ministerio de Salud decreta la creación del "Comité de Investigaciones Médicas en Humanos” y reglamenta los ensayos de nuevas drogas y medicamentos mediante el decreto ejecutivo 2393. (1) Este hecho presagiaba un desarrollo normativo riguroso en este campo, que como se verá más adelante, no sucedió. Luego, en el año 1973, se aprobó la Ley General de Salud (2), vigente desde entonces, incluyendo algunas regulaciones generales sobre investigación con seres humanos. (Bustos, 2007, p. 40)

Lo anterior muestra que un importante objetivo, relacionado con la investigación médica, fue el nexo entre quien investiga (generalmente el personal médico) y la persona investigada (el sujeto), lo cual llevaba, de cierta manera, a los mismos problemas éticos existentes entre el médico y el así llamado paciente: paternalismo, autoridad, relaciones de poder, conocimiento esotérico por parte del médico y poca autonomía por parte del paciente. Esto podría ser suficiente para una ética que regulara comportamientos y que, desde el punto de vista normativo, pudiera aplicar sanciones a las faltas en la práctica

22 Es el primer código después de la Segunda Guerra Mundial que se crea con el fin de proteger la vida humana de la experimentación. Desde entonces se han propuesto más, para velar por la seguridad de los sujetos, convirtiéndose en códigos éticos. Por ejemplo, el primer punto de este contempla aspectos de autonomía, y el segundo habla sobre el beneficio a la sociedad, que es conocido hoy como el principio de beneficencia. Este es de vital importancia para discutir los alcances éticos del expediente digital único en salud EDUS.

23 Una de las primeras investigaciones realizadas en Costa Rica -al menos de las documentadas- fue realizada en 1959 por la Unidad de Estudios de Poliomielitis de la Universidad de Yale. Para este se reclutaron 48 familias de Santo Domingo de Heredia (Horstman et. al., 1961). 
médica ${ }^{24}$. Dada la situación económica de Costa Rica en la segunda mitad del siglo XX, hasta más o menos 1997, tal como se mencionó, la actividad médica era prácticamente asistencial. No eran de dominio médico muchos de los avances tecnológicos y las tecnologías de información, en otras palabras, hacia la misma época que se crean tanto la CCSS como la Facultad de Medicina de la UCR (década de los 40 del siglo XX) estas no tenían impacto directo.

$\mathrm{Al}$ retomar los problemas éticos, asociados a las nuevas tecnologías en el país, cabe señalar que una cosa es apropiarse de tecnologías y otra crear y diseñar las propias. Este es un tema relacionado con la forma en la que se asume la investigación científica y tecnológica en los países "en vías de desarrollo". Las personas suelen tardar más tiempo en adaptarse a nuevas tecnologías en un país que no las generó; pueden oponerse más a aplicarlas que las de los países originarios. Ejemplo de ello es la técnica de reproducción asistida llamada Fertilización in Vitro, que desde 1978 aparece en países como Estados Unidos, y que fue prohibida en Costa Rica en el año 2000, y, 17 años después por medio de un fallo de la Corte Interamericana de Derechos Humanos se logró implementar dicha técnica en la CCSS.

\section{Tecnología y salud: los datos médicos y el paciente.}

Cuando se habla de tecnología, nos hemos acostumbrado a palabras como computadoras, teléfonos y pantallas inteligentes, drones y un sinfín de artículos que han inundado de manera reciente nuestras vidas. Sin embargo, la medicina ha empezado a utilizar la tecnología, en particular, la desarrollada a través de la inteligencia artificial (IA), para realizar operaciones como las llamadas cirugías robóticas ${ }^{25}$. En un artículo del diario El Financiero, del 31 de

24 No es objetivo de este estudio dar a entender que estos problemas están superados, al contrario, prueba de ello fue la condena en el mes de enero de 2018 a un médico del Hospital Calderón Guardia, acusado por tráfico de órganos. Sin embargo, lo que se quiere señalar es que los problemas en medicina ahora son mucho más complejos de lo que eran hace unos 70 años. Por ejemplo, los problemas asociados a la FIV, la manipulación genética o, más recientemente, la clonación de dos macacos por parte de científicos de China.

25 Tal como se mencionó en el punto $\$ 1.2$, los accidentes médicos relacionados con la tecnología han sido pocos, teniéndose como el principal, el de irradiados, sucedido en el Hospital San Juan de Dios. No obstante, hay que acotar que también la definición de accidente tecnológico para el Ministerio de Salud no parece ser la adecuada. Por ejemplo, en el documento "Emergencias Tecnológicas en Costa Rica 2001-2002”, lo que se considera una emergencia tecnológica tiene que ver más con accidentes relacionados con sustancias químicas, y no eventos causados por dispositivos tales como computadoras, robots, software, etc. Un reporte del uso de cirugías robóticas, llamado "Adverse Events in Robotic Surgery: A Retrospective Study of 14 Years of FDA Data" asegura que, en Estados Unidos se han producido 144 muertes por el uso de estas tecnologías en el ámbito médico. En la 
octubre del año 2017 ya se mencionaba la incursión de estas tecnologías en el país, principalmente en el sector médico privado, tanto en la UNIBE como en la Clínica Católica ${ }^{26}$. Tal como sucedió con el expediente digital, también en el sector privado se implementan, en algunas ocasiones, nuevas tecnologías. Al parecer, la CCSS no tiene planes aún de introducir algunas como el bisturí virtual, los robots que simulan y otros tantos equipos con inteligencia artificial en la atención a los usuarios.

Ahora, ¿por qué investigar e invertir en salud desde la inteligencia artificial?27. La respuesta parece ser obvia: los datos -y particularmente en este contexto los datos de salud-son una mina de oro ${ }^{28}$. Cualquier sistema informático necesita alimentarse de datos, y para que un sistema de este tipo pueda funcionar deben cumplirse al menos tres condiciones:

1) que los datos estén disponibles para ser procesados por un sistema informático 29

2) que los datos estén en un formato adecuado

3) que estos no tengan restricciones de uso o sean mínimas

Con respecto al primer punto, la implementación del EDUS permite la disponibilidad de los datos. De hecho, empresas como Cognitiva justifican la necesidad de la IA en el sector salud, basándose en la necesidad de un "eficaz" manejo de estos. Pero, tal como se mencionó precedentemente, no hay un correcto análisis crítico del producto y se compra como si fuera un elemento más, que permite un paso acelerado hacia el desarrollo, basado en la tecnología. En este sentido, los profesionales en informática necesitan generar un pensamiento crítico y ético ante la recepción de tecnología extranjera -proveniente de

actualidad no hay regulaciones ni reflexiones sobre cómo proceder con esta problemática en Costa Rica, y esto no podría considerarse desde el punto de vista bioético simplemente como un "evento adverso".

26 Carlos Cordero señala que en la Clínica Católica se utilizan elementos de IA en el diagnóstico y atención al usuario del sistema de salud: "Es el caso de un angiógrafo, un equipo especializado para realizar cateterismos cardiacos" (El Financiero, 31 octubre, 2017, párrafo 12).

27 De hecho, uno de los eslogans de Cognitiva, empresa latinoamericana subsidiria de IBM y representante del software Watson es "La computación cognitiva va a cambiar la forma en la que opera el área de la salud". (https:// www.cognitiva.la/industrias/salud/), pero para esto, se ocupan datos.

28 Este tema será analizado un poco más adelante, aunque no deja de ser extraño que un sistema como Watson, con varios años de funcionamiento incursione en Costa Rica en el 2017, cuando el expediente digital en salud comienza a ser más sólido en los tres niveles de atención de la CCSS.

29 Es de notar que, en marzo de 2018, de acuerdo con Manuel Rodríguez Arce, gerente informático del proyecto EDUS, el total de los datos almacenados respecto de la salud de la población era de 1 Tera, esto es, 1000000000000 bits, aproximadamente 4.5 millones de libros de 200 páginas. 
países desarrollados-.En otras palabras, se sugiere la centralizar la información médica apelando a categorías como eficacia, producción y desempeño, y se invoca, por supuesto, el bienestar del paciente (Financiero, 15 octubre, 2017). No obstante, esta ética utilitarista, aplicada a la tecnología, ve simplemente al usuario como un medio, y no como un fin. Después de todo, esto es costoso ${ }^{30}$ y por eso no parece ser parte de la gama de servicios médicos, que por ahora, puede ofrecer la CCSS a la totalidad de la población asegurada.

Acerca del segundo punto, implementar el EDUS permite que los datos estén en un formato adecuado para el análisis, esto es, si ya no están en un documento como el expediente físico ${ }^{31}$, el poder alimentar a tal sistema se vuelve mucho más fácil, porque la información se reduce básicamente a unos y ceros, como suelen ser los lenguajes en los que se programa. De esta manera, para empresas como Cognitiva, o para sistemas que tratan de aplicar conocimientos de IA en medicina, es más fácil el uso de datos codificada que pueda procesarse por lenguajes no naturales. En este sentido, el EDUS representa una fuente de información disponible, solo se necesita el canal o los medios adecuados para tener acceso a ella ${ }^{32}$.

En cuanto al tercer punto, proteger los datos, parece al menos, estar garantizado por la Ley N. o 8968 del año 2011, o Ley de Protección de la Persona frente al Tratamiento de sus Datos Personales. Esta ya tiene casi 7 años de haberse publicado, y los cambios que ha experimentado el mundo parecieran hacer obsoleta la normativa que debe regir para el uso de datos. Por ejemplo, si bien es cierto que la ley describe con algún nivel de detalle lo que es una base de datos ${ }^{33}$, no especifica los límites para su administración.

30 Por ejemplo, solo la inversión en el EDUS podría llegar a la cifra de $\$ 100$ millones (La Nación, 30 de mayo, 2015), lo cual demuestra que la tecnología no es barata, es costosa de aplicar y su conocimiento, contrario a lo que sucede con el llamado software libre.

31 Lo cual lleva a otra reflexión. El trabajo de los archivistas es ahora realizado por el encargado de bases de datos, que generalmente es un profesional en informática.

32 No deja de ser curioso en este contexto que el programa del Partido Acción Ciudadana en algún momento mencionara la posibilidad de compartir la información médica con empresas privadas: "Creación de un Centro Nacional de Investigación en Tecnologías Alternativas de Saneamiento para Integrar al sector estatal, comunal, privado, científico y académico con el fin de desarrollar nuevas tecnologías de saneamiento priorizando las zonas de mayor vulnerabilidad." (Programa de Gobierno 2018, PAC, p. 148), esto demuestra lo útil que pueden ser los datos, particularmente en salud, lo cual sugiere el problema ético con el manejo y privacidad.

33 La ley da dos definiciones importantes: a) Base de datos: cualquier archivo, fichero, registro u otro conjunto estructurado de datos personales, que sean objeto de tratamiento o procesamiento, automatizado o manuales, cualquiera que sea la modalidad de su elaboración, organización o acceso. b) Datos personales: cualquier dato relativo a una persona física identificada o identificable. Y establece una categoría llamada e) Datos sensibles: información relativa al fuero íntimo de la persona, como por ejemplo los que revelen origen racial, opiniones 
Cabe preguntarse en qué lugar están ubicados los servidores, cuáles son los niveles de seguridad mínimos y quién se encargará de supervisar estos elementos ${ }^{34}$. De hecho, con la firma de un consentimiento informado cualquier compañía podría acceder a los datos de las personas, almacenados electrónicamente, por lo que existe un portillo en la ley gracias al cual la CCSS podría compartir esos datos con terceros. En efecto, la ley establece lo siguiente "Quien recopile datos personales deberá obtener el consentimiento expreso de la persona titular de los datos o de su representante. Este consentimiento deberá constar por escrito, ya sea en un documento físico o electrónico, el cual podrá ser revocado de la misma forma, sin efecto retroactivo”. (inciso 2 , artículo 5, Ley N. o 8968)

Pero hay una pregunta que no parece tener respuesta clara: ¿a quién le pertenecen los datos médicos de los asegurados de la CCSS, al usuario o a la CCSS? A manera de introducción para un análisis ético de un problema informático, relacionado con bases de datos, en el 2016, entre marzo y mayo (Financiero, 26 de mayo, 2016) se realizó un ataque ${ }^{35}$ a las bases de la CCSS, que resultaba en robo de la información salarial de 522000 asegurados. A la fecha no se han sentado responsabilidades, lo que demuestra los vacíos legales existentes en materia de datos informáticos. De acuerdo con la CCSS "hubo una violación al derecho de auto determinación informativa de los usuarios de la Caja Costarricense de Seguro Social” (El Financiero, 26 de mayo de 2016, párrafo 17). Esto resulta una violación al artículo 3 de la Ley 8968, pero a pesar de ello no se ha tomado ninguna decisión adecuada para salvaguardar datos y definir los usos que puedan darse. Esto nos hace pensar en uno de los

políticas, convicciones religiosas o espirituales, condición socioeconómica, información biomédica o genética, vida y orientación sexual, entre otros. No obstante, el reglamento emitido por la CCSS sobre el expediente digital en salud no especifica si los datos en salud de las personas son: sensibles, personales, personales de acceso irrestricto o datos personales de acceso restringido.

34 Tampoco existe un proceso para fiscalizar si la información se guarda en servidores en la nube o no, sin embargo, en la directriz 049-H el Gobierno de Costa Rica ha solicitado a todas las instituciones del sector público estar en la nube. (http://www.digeca.go.cr/sites/default/files/directriz_46-h-micitt-la_gaceta_93-16_mayo_2013_tecnologias_de_informacion.pdf) Para comprender de lo que es computación en la nube, ver anexo 1 .

35 No quiere decir que haya sido el único, de acuerdo con datos proporcionados por Robert Picado, de la Subgerencia de Tecnología y Comunicaciones de la CCSS, "desde el hackeo al Sicere, se han realizado mejoras a la infraestructura de seguridad en tecnologías de la información (TI). Con esto, se bloquean, en promedio, 35.350.022 ataques de seguridad informática al mes. Se trata de intentos que son bloqueados cada segundo las 24 horas, los 365 días al año. De esos, 691.027 son ataques de severidad alta (2\%), y 34.658.995 son de severidad media (98\%)", (La Nación, 4 de setiembre de 2016, párrafo 13) 
actores principales: el usuario del sistema de salud y su involucramiento en la toma de decisiones de las instituciones estatales que velan por su bienestar.

\section{El acceso a internet.}

Una nota del diario digital El Mundo CR, del 24 de marzo de 2015, indica que solo la mitad de la población costarricense tiene acceso a internet:

Menos de la mitad de la población costarricense tiene acceso a Internet, según reveló el último informe de la Comisión de Banda Ancha de las Naciones Unidas. De acuerdo con el estudio, en el país solo $46 \%$ de la población tiene conectividad a Internet, quedando en la posición 84 de 191 países estudiados. (elmundocr., 24 de marzo de 2015, párrafo 1)

Si esto es así, se da un alto grado de vulnerabilidad para casi la mitad de la población costarricense que no tiene dispositivos móviles para acceder a información de su expediente digital. Asimismo, si el uso de internet está limitado a la mitad de los usuarios debería analizarse también el nivel de acceso a las computadoras por parte de esta. Habría que hacer también un análisis de cómo las personas perciben esta herramienta implementada por la CCSS, pues podría no haber claridad de cómo sus datos están siendo manipulados tecnológicamente. Esto, por supuesto, nos lleva a cuestionarnos, bajo otra perspectiva, la relación del paciente con los datos generados por el EDUS.

Por otra parte, el tratamiento basado en el diagnóstico escrito por el médico y la necesidad del usuario de saber sobre su salud llevan a un nuevo debate sobre el paternalismo médico en la era tecnológica ${ }^{36}$. Se podría pensar, entonces, tanto en las limitaciones que imponen los profesionales en salud para que un usuario pueda ver sus datos médicos y en las herramientas tecnológicas con las que cuenta una población que no está utilizando en su totalidad internet o las tecnologías de información. Datos publicados en el periódico CRhoy.com dice que:

36 Una encuesta de Harris-Polls, del 2013, reveló los siguientes datos: 82 \% of U.S. physicians want patients to update their electronic health records with information about themselves, but only $31 \%$ believe patients should have full access to that record; $65 \%$ believe patients should have only limited access. Four percent said patients should have no access at all. Esto fue hecho con base en las respuestas de 3700 médicos de ocho países. (https:// www.computerworld.com/article/2496084/u-s-doctors-don-t-believe-patients-need-full-access-to-health-records. html) 
Según Sutel, en el país hay más de 8 millones de líneas celulares inscritas. Un 65.3\% de ellas son prepago. "En telefonía móvil, Costa Rica tiene una penetración de 179 líneas celulares por cada 100 personas. Eso quiere decir qué hay casi 2 teléfonos por persona”, ejemplificó la superintendencia (CRhoy, junio, 2018, 19 de junio, párrafo, 4).

Según datos de la CCSS, las descargas de la aplicación móvil al 23 de enero de 2018 eran: Android= 228 960, IOS= 13 437, para un total de: 242 397 (Fuente, CCSS, Ing. Miguel Arce).

Esto nos muestra un nivel muy bajo, cercano al $3 \%$ de la población del país, en el uso de la aplicación del EDUS por medio del teléfono celular, para acceder a la información de su propio expediente. Si a esto le agregamos que el $63 \%$ de las personas usan su teléfono con el sistema de prepago, podría pensarse que estas no van a gastarlo en una aplicación como el EDUS. Existe, además, un problema de género, ya que muchas mujeres ${ }^{37}$ son jefas de hogar y les es más barato financiar una conexión de tipo prepago que una fija de internet, esto por no hablar de las diferencias salariales existentes entre hombres y mujeres. La apuesta por una salud en línea es de admirar, pero es necesario analizar con el tiempo la información que permita determinar el impacto en la salud de las personas por la implementación del expediente digital versus el impacto económico en la CCSS.

\section{Referencias}

Alemzadeh, H., Raman, J., Leveson, N., Kalbarczyk, Z., \& Iyer, R. K. (2016). Adverse Events in Robotic Surgery: A Retrospective Study of 14 Years of FDA Data. PloS one, 11(4), e0151470. doi:10.1371/journal.pone.0151470

Arenales, M. A. (2002). Visitas al desvan . San José: Alambique.

BIBLIOGRAPHY Avalos, A. (2016, setiembre 4). 'Hackeo' a base de datos de CCSS en la impunidad. La Nación.

Bíblica, H. C. (2012). Innovadora Solución. Por su salud, 12-14.

37 The International Telecommunications Union reports that the proportion of women using the internet is $12 \%$ lower than the proportion of men; this gender gap widens to $32.9 \%$ in the least developed countries. And even when a woman gets on a phone or is online, she might face additional hostility. A World Wide Web Foundation report says "women around the world report being bombarded by a culture of misogyny online, including aggressive, often sexualized hate speech, direct threats of violence, harassment, and revenge porn involving use of personal/private information for defamation" (Web Foundation, 2015). 
Clinfowiki. (2015). Computer Stored Ambulatory Record (COSTAR). Recuperado el 13 de julio de 2017, http://www.clinfowiki.org/wiki/index.php/ COmputer_STored_Ambulatory_Record_\%28COSTAR\%29

Cordero, C. (2017, octubre 31). La inteligencia artificial ya brinda asistencia médica en Costa Rica. El financiero.

Cruz, M. F. (8 de febrero de 2015). Expediente Digital Único de Salud, una mina de datos que enfrenta los retos de su propio contenido. El Financiero.

Editorial. (2015, mayo 30). El laberiton del expediente digital. La Nación.

Foundation, W. (2015). Five barriers, five solutions: Closing the gender gap in ICT policy. Washington D.C. : Web Foundation .

Legislativa, A. (2011, julio 7). Protección de la Persona frente al tratamiento de sus datos

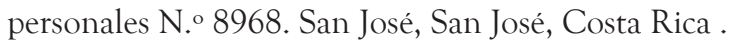

Mead, L. M. (1896). Beyond Entitlement. New York: Simon \& Schuster .

Mearian, L. (2013, marzo 7). U.S. doctors don't believe patients need full access to health records. Computer World.

MICITT. (2013, mayo 16). DIRECTRIZ N. * 46-H-MICITT. La Gaceta.

Monteiro*, D. B. (2007). Evolución de la bioética en Costa Rica: una historia reciente. Bioetikos, 39-44.

PAC. (2017, mayo 15). Plan Gobierno 2018-2022. San José, San José, Costa Rica.

Paul, J. R., Horstmann, D. M., Riordan, J. T., Opton, E. M., Niederman, J. C., Isacson, E. P., Green, R. H. (1962). An oral poliovirus vaccine trial in Costa Rica. Bull World Health Organ, 311-329.

Picado, F. (2016). Nuevas tecnologías de información aplicadas en salud. En C. C. Social, Metamorfosis 2041. San Jose.

Rojas, P. (2018, junio 19). Telefonía móvil en Costa Rica: hay casi 2 líneas celulares por persona. CR Hoy .

Rodríguez, M. (2018 de marzo de 2018). Aspectos técnicos del EDUS. (A. Gonzalez, Entrevistador)

Shop, R. (2013). History Of Electronic Medical Records History Of Electronic Medical Records. Recuperado el 15 de octubre de 2017, http://www.healthtechnologyreview.com/ art135_history_of_electronic_medical_records.php

Singleton, P. (2009). Critical Issues for Electronic Health Records. Nuffield Council of Bioethic. Nuffield Council of Bioethics.

The Secretary of State for Culture, M. A. (2009). Digital Britania. Recuperado el 12 de octubre de 2017, dhttps://www.gov.uk/government/uploads/system/uploads/attachment_data/file/228844/7650.pdf

Universal health data in Costa Rica: The potential for surveillance from a human rights perspective. (2014). Recuperado el 19 de diciembre de 2017, https://www.giswatch.org/sites/ default/files/universal_health_data_in_costa_rica.pdf

Varela, J. C. (2017). Análisis de la implementación del Expediente Digital Único en Salud y su impacto en el cumplimiento de las metas en el Primer Nivel de Atención del Área de Salud Buenos Aires durante el año 2016. Recuperado el 5 de enero de 2018, http://biblioteca.icap.ac.cr/ BLIVI/TESIS/2017/91.\%20Calderon,\%20Jorge\%20.pdf 